\title{
Les manuels de lecture du cours élémentaire, lieu romanesque et instance de la moralisation scolaire
}

\section{Nicole Bourdon}

\section{(2) OpenEdition \\ 12 Journals}

Édition électronique

URL : http://journals.openedition.org/trema/1802

DOI : 10.4000/trema.1802

ISSN : 2107-0997

Éditeur

Faculté d'Éducation de l'université de Montpellier

\section{Édition imprimée}

Date de publication : 1 décembre 1998

Pagination : 27-39

ISSN : 1167-315X

\section{Référence électronique}

Nicole Bourdon, «Les manuels de lecture du cours élémentaire, lieu romanesque et instance de la moralisation scolaire », Tréma [En ligne], 14 | 1998, mis en ligne le 01 décembre 1998, consulté le 01 mai 2019. URL : http://journals.openedition.org/trema/1802 ; DOI : 10.4000/trema.1802

Ce document a été généré automatiquement le 1 mai 2019.

Trema 


\title{
Les manuels de lecture du cours élémentaire, lieu romanesque et instance de la moralisation scolaire ${ }^{1}$
}

\author{
Nicole Bourdon
}

1 Les anecdotes brûlantes sur la violence à l'école, dont nous abreuvent les médias aujourd'hui, témoignent d'un profond malaise dans notre société. Ce phénomène nous a conduit à réfléchir sur le rôle de l'école dans le développement moral de l'enfant, à travers le principal vecteur de la transmission du savoir: le manuel scolaire. Outil pédagogique, support de connaissance, fonds commun de référence identitaire, le manuel scolaire est un objet fortement idéologique, qui diffuse, de façon plus ou moins explicite, des valeurs d'ordre éthique, social ou scolaire.

2 C'est sur ce dernier point que nous nous proposons d'axer notre recherche, à travers l'analyse des manuels de lecture de C.E.1 des années 1930, 1950, 1980².

Mises bout à bout et traitées synthétiquement, les brèves séquences de la vie scolaire, transcrites à travers les livres de lecture de notre corpus, finissent par former un film sur l'école. Cette chronique familière reflète une certaine image de ce lieu où, par l'entremise de l'instituteur, le personnage de l'enfant originel, encore proche de l'état de nature, abandonne son égoïsme individuel pour se transformer en un écolier responsable apte à vivre en société.

Figure 1

L'école primaire, modèle 1930.

image

MIRONNEAU A. : Choix de lectures - Cours Élémentaire. Paris, A. Colin, 1930, 232 p., p. 53. 


\section{A l'école !}

\subsection{Le scénario des années 1930}

3 Nous sommes le 30 septembre, à la veille de la rentrée des classes. Parce que l'école est l'affaire de tous, la famille est réunie au grand complet dans la salle à manger : le père, la mère et leurs enfants, Francine et Pierre. Deux serviettes d'écoliers, posées sur la table, annoncent le grand jour [6]...

Francine, Pierre, Alain, Tifernand et les autres sont âgés de sept, huit ou neuf ans. Ils sont de caractère et de tempérament différents. Certains habitent la campagne, d'autres la ville. Tous ont revêtu, ce matin, leurs beaux habits et s'apprêtent à vivre leur premier jour de classe.

Ce premier jour d'automne est beau comme un jour d'été. Les oiseaux chantent. Quel temps splendide! [2] Les enfants se sont-ils soigneusement lavés? Ne leur manque-t-il rien? Avec des sentiments d'amour et de fierté, monsieur et madame Devarenne contemplent leurs enfants : une nouvelle vie les attend, aussi courent-ils, riant et joyeux comme s'ils craignaient de ne pas arriver assez tôt à l'école [6].

4 Francine s'est levée de bon matin [4], elle a mis sa robe du dimanche et préparé consciencieusement son cartable. Sur le chemin de l'école, elle sent bien qu'elle n'est plus ce matin une toute petite fille, et que quelque chose de grave commence pour elle [3]. Pierre, quant à lui, le béret sur la tête et le sac à l'épaule, marche ferme et droit, martial comme un vaillant conscrit, d'un pas assuré, sans perdre de temps comme il se doit [1]:

"Où va-t-il ce bambin pas plus haut qu'une botte?

Il a mis ce matin sa plus belle culotte;

Rose et frais, bien peigné, dans son habit propet,

Le voilà dans la rue, alerte et guilleret. » [1]

Le cœur battant, Francine et Pierre s'approchent de la maison grise aux grandes fenêtres, et se dirigent vers la grille de la cour d'école, Francine non sans appréhension [5], et Pierre sans faillir [1]. Ils reconnaissent des petits camarades qui forment une ronde en chantant, mais leurs chansons sont sages comme celles des oiseaux en cage [5]. Tous ont bonne mine et leur disent bonjour d'un air joyeux [4]. Prévenue de leur arrivée, la maitresse les accueille à bras ouverts. Elle a une voix douce et agréable [6].

Soudain, la cloche sonne. Les jeux et les cris s'arrêtent. Les enfants se rangent devant la porte de la classe. Quelques petits bavardent encore un instant puis se taisent eux aussi dans la cour muette. Ils passent devant Mademoiselle les mains dans le dos et entrent en bon ordre en chantant une jolie chanson [5].

Fauvette est fort troublée [7]; après un gros soupir, elle s'assied, croise ses bras sur la table et regarde autour d'elle, un peu craintive [5]. Une fillette se retourne vers elle avec un sourire d'amitié, mais Fauvette n'a pas le cœur à répondre à ce sourire [3].

Un coup de règle frappé sur une table la fait sursauter et une voix sévère dit : "Silence! Silence!» [5]La nouvelle maîtresse a l'air un peu grave dans sa robe noire mais Fauvette comprend que ce visage est obligé de dissimuler sa vraie bonté, et elle en est réconfortée [7].

6 Mademoiselle Claire distribue des livres aux grands élèves et des bâtonnets aux plus petits. Les premiers récitent des fables que la maîtresse leur a expliquées, ils lisent, comptent, écrivent, apprennent l'orthographe cependant que les plus petits regardent de 
belles gravures instructives [2]. Chariot tire gravement son syllabaire du cartable. Il fronce son petit front et s'applique à bien lire [2]. Fauvette travaille maintenant de tout son cœur, elle entend faire plaisir à sa maman et, surtout, elle veut mériter un sourire ou un compliment de Mademoiselle [3].

La maîtresse pose une question. Une fillette ou un garçonnet se lève et balbutie une réponse, tête baissée [5]. Vigilante, Mademoiselle Claire ne quitte pas des yeux les élèves et reprend de temps en temps les distraits : «Vous ne suivez pas!» [7]Marcel et Louis sont contraints de rectifier leur attitude car au lieu d'écouter les leçons, ils bavardent sans cesse et dérangent la classe [8].

7 Mais de nouveau, la baguette sévère commande le silence. Les élèves ont le dos baissé et s'appliquent à leurs besognes [5]. Enfin la maitresse demande de ranger les livres et les cahiers. Les enfants sortent en rang et, à un nouveau signal, se dispersent dans la cour [9].

"Je lis dans vos yeux satisfaits,

que vous avez eu du courage,

Et que vos devoirs sont bien faits:

Cour content fait joyeux visage » [5].

\subsection{Le scénario des années 1950}

"C'est l'heure de la classe, a dit la mère, en route!

Les yeux pleins de sommeil, les petits écoliers

S'habillent à tâtons, mettent leurs gros souliers

Et les voilà partis, grignotant une croûte !... » [19]

C'est un beau jour que celui de la rentrée, presqu'un jour de fête. Dans la rue, les écoliers s'interpellent et se disent bonjour. Ils sont propres et soignés avec des tabliers neufs, un peu raides. Tous se hâtent vers l'école [20].

Les moins timides vont dire bonjour à leur maître. Quelques fillettes apportent des fleurs à leur maitresse qui les a embrassées [20]. Son panier sous le bras, son cartable sur le dos, Tifernand arrive dans la cour, traînant les pieds et sifflotant. Que ce maître a l'air féroce ! Que cette maîtresse a l'air méchant [16] ! Puis survient Alain, un petit nouveau, la joue rose, l'œil brillant [15], bien peigné, bien lustré, tout plein du désir d'apprendre [11]. Ses parents l'accompagnent jusqu'à la grille de l'école [13].

La cloche annonce l'entrée de la classe. Les élèves se mettent en rang, sans désordre, et entrent dans la salle après la visite de propreté. Comme l'école est belle ce matin, tout reluit, tout brille [20]!

9 Le vieux maître de la classe des grands trempe sa plume dans l'encre et annonce qu'il va faire l'appel. A l'appel de son nom, chaque élève se place à la table désignée par le maître [13]. De beaux cahiers, des livres neufs reposent sur les pupitres [20].

Dans la salle voisine, Mademoiselle Lefort fait de même avec ses élèves. Sa voix est douce et agréable. Elle n'a pas besoin de parler fort, chacun sait ce qu'il doit faire [20].

Une formidable gaîté se déchaîne sur les bancs. La douceur de cette demoiselle et la joie des enfants inspirent confiance à Tifernand [13]. Il se sent un peu prisonnier, encagé entre les quatre murs de la classe. Mais, il faut bien retourner à l'école après plus de deux mois de vacances [15].

Dans la voisine, la maitresse frappe un petit coup de règle sur le bureau. Toutes les têtes se lèvent. "Mes enfants", dit-elle, "nous commençons ensemble une nouvelle année. Il faut qu'elle soit bonne. Vous me promettez, n'est-ce-pas, de travailler de tout votre cœur? „Tous les yeux brillent et toutes les bouches répondent « oui» [20]. 
11 Le maître du Cours préparatoire tient en main une baguette qu'il promène sur le tableau noir, et chaque fois que le bout de la baguette s'arrête, tous les écoliers se mettent à répéter ensemble les lettres de l'alphabet [17].

Puis, chacun s'applique à écrire son nom, de la plus belle écriture [20]. Les garçonnets et les fillettes sont sagement penchés sur leur pupitre et ne quittent pas des yeux la page étalée devant eux [17]. Le maître passe dans les rangs et donne une petite tape affectueuse aux plus appliqués [20].

Pendant la classe, Alain ne bavarde jamais, ni ne fait aucun bruit. C'est un très bon élève, attentif et appliqué. Quand il regarde sa petite camarade, Suzette, il lui sourit des yeux, comme pour lui dire "nous sommes amis " [20]. Elle aussi est très appliquée et rêve de devenir institutrice. Elle s'imagine déjà, devant le tableau noir, le bâton de craie à la main, répondre au nom de Mademoiselle. Quel beau métier ce sera ! pense-t-elle [21].

Le directeur sonne une petite cloche. Tous les écoliers rentrent à la maison. Restent, après quatre heures, les punis de toutes les classes qui se réunissent dans une salle. Tifernand est l'un d'eux [14]. Pendant la classe, il n'a cessé de rire, de faire des grimaces à sa voisine donnant ainsi toutes sortes de signes de son insouciance et de son espièglerie [13].

Figure 2

L'école primaire, modèle 1950.

image

BRÉANT L.-M. et THIERRY B. : Nous les enfants. Paris, A. Colin, 1955, 159 p., p. 23.

14 Maintenant, il n'est pas fier et attend avec angoisse l'appel de son nom auquel il répond d'une voix étranglée et faible [14].

Puis, il revient de l'école, la tête basse. Il marche vite sans s'amuser de tout et de rien, comme à son habitude. Etre puni le premier jour de classe, ce n'est pas reluisant [15].

Alain, lui, est rentré à la maison plein d'allégresse, il est satisfait de montrer son carnet de notes à son grand-père qui prend plaisir à le feuilleter et à lire à haute voix les appréciations élogieuses : Bon petit élève, appliqué et travailleur [15].

"Nous marchions d'un pas décidé et claquant

Nous pépiions comme une bande de moineaux.

Notre voisine nous faisait signe et disait :

Sont-ils mignons ainsi ! Sont-ils gentils!» [16]

\subsection{Le scénario des années 1980}

Nous voici parvenus aux années 1980. Le regard sur l'école perd de son objectivité : il épouse le point de vue de l'enfant et adopte désormais la première personne.

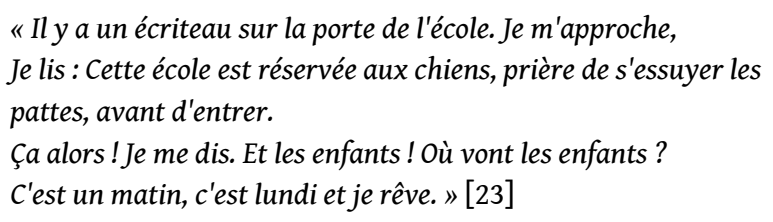

17 L'horloge sonne, c'est bientôt l'heure de la rentrée des classes, il faut faire attention de ne pas arriver en retard [24]. Je suis tout fier d'étrenner mon nouveau cartable; sur le chemin de l'école, je rencontre mon copain Andy [26] qui est beaucoup moins enthousiaste que moi. Il a le cafard [31]. 
Chemin faisant, je pense que, lorsque je serai plus grand, «je serai vaillant comme un chevalier... » [22]. Je me presse pour arriver avant la maîtresse qui traverse le préau, son attaché-case marron à la main [29]. L'école est accueillante. La cour de récréation est pleine d'enfants turbulents habillés de toutes les couleurs [28]. Comme chaque jour, la bande des grands s'est regroupée sous les platanes [29].

Tous les élèves rentrent dans les classes. Andy a envie de courir vers les vestiaires mais il respecte la règle : on ne doit pas courir dans les couloirs [31]. "Bonjour les enfants ", dit la maîtresse, "entrez et asseyez-vous! Revoyons la leçon de calcul» [24]. De beaux crayons feutres de couleur nous attendent sur leur table pour nous aider à comprendre les mathématiques [28].

Madame Tournois interroge Andy qui ne sait pas répondre. Il se fait gronder car il n'écoute pas en classe et ne sait pas calculer. La classe récite en chœur :

"Deux et deux font quatre

quatre et quatre font huit

huit et huit font seize

Répétez, dit le maître. " [31]

Puis la maîtresse regarde ses mains et prend un air gêné en disant : «Mon pauvre Andy, tes mains sont bien sales. Et les ongles, fais voir! Eh bien dis donc, il faut que tu te laves. "Les autres élèves rient et Andy se sent tout confus [31].Pourtant il essaye de faire des efforts, aussi trouve-t-il ces reproches injustes, il regrette sa maîtresse de l'année dernière qui souriait tout le temps [31].

Figure 3

L'école primaire, modèle 1980.

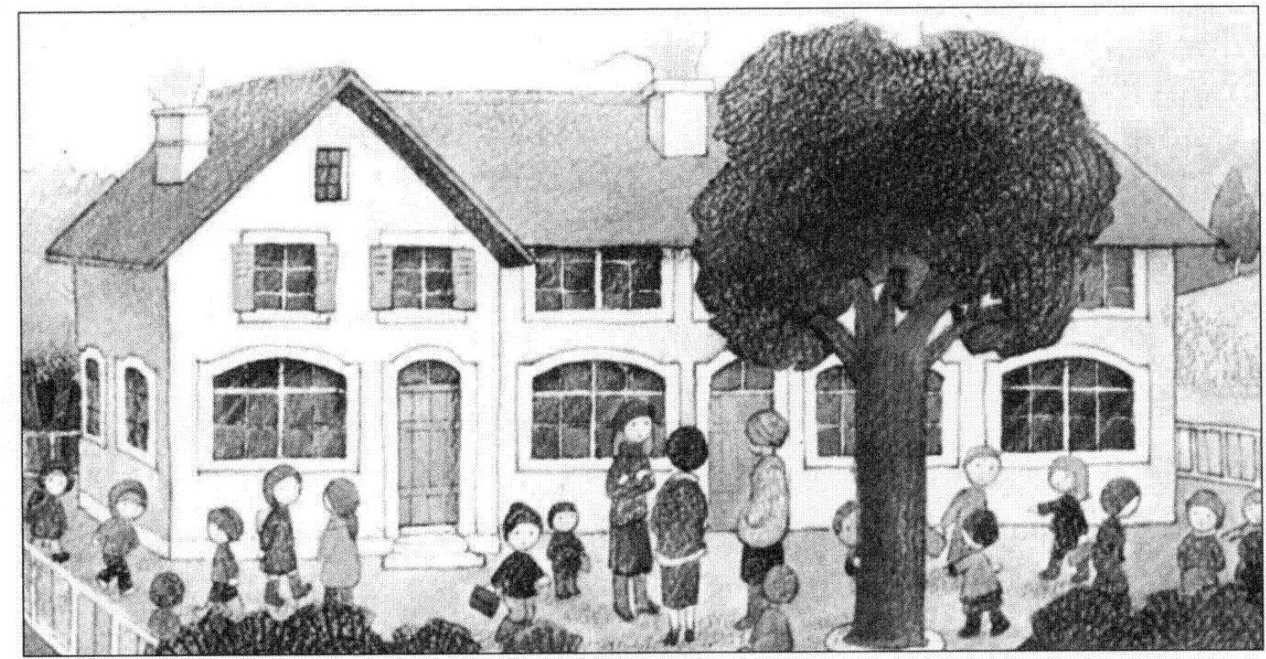

KERLOC'H J.-P. : La planète des livres. Paris, Bourrelier, A. Colin, 1984, 127 p, p. 11.

Ma copine Julie est sérieuse, elle sait que pour ne pas tout oublier, il faut travailler régulièrement [24]. Quant à moi, je suis persuadée que la maîtresse est une fée. Je suis émerveillée par sa rapidité à corriger les cahiers alors même que, dans la journée, on fait des tas d'exercices et de problèmes : le lendemain, sans faute, nous retrouvons tous nos devoirs soigneusement notés, avec de petits mots personnalisés pour chacun [30]. Et puis, j'aime aussi quand la maîtresse nous soigne : si l'un de nous a une bosse, elle pose sa main dessus, appuie fort en fermant les yeux, et la bosse disparaît comme par magie; si 
quelqu'un saigne du nez, elle lui pince le cou, lui tapote le dos et le saignement s'arrête. Ma maîtresse dit que c'est de la médecine japonaise [30].

Juste avant la pause, la maîtresse fait une projection de diapositives et nous incite à ouvrir les yeux et à regarder autour de nous pour découvrir les belles choses que la nature peut offrir [30]. Puis, silencieusement, nous nous mettons en rang dans le couloir [31].

Pendant la récréation, le maître du Cours Élémentaire surveille les élèves qui jouent bruyamment dans la cour ; sous le tilleul, la maitresse du Cours Moyen bavarde avec celle du Cours Préparatoire, et on aperçoit le directeur de l'école dans son bureau qui reçoit les parents [27].

L'école finit à cinq heures. Je me dépêche de rentrer à la maison. En arrivant, comme à mon habitude, après une journée bien remplie, je pose mon cartable et me déchausse [25].

\section{Image et fonction de l'école}

\subsection{Un lieu d'instruction}

L'école primaire a pour fonction première d'enseigner la lecture, l'écriture, l'orthographe et le calcul : autant de connaissances correspondant à des savoir-faire pratiques, car il ne faut pas oublier que, dans l'ensemble - du moins jusqu'à l'institution de la scolarité jusqu'à seize ans - l'entrée dans le monde du travail et l'accession à l'état d'adulte sont beaucoup plus précoces que de nos jours.

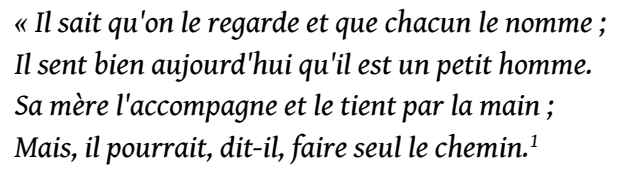

Dans un contexte de plein emploi, où la possession du Certificat d'Études assure un métier, l'école bénéficie de la confiance générale de la population. Par ailleurs, dans la mesure où elle est l'instrument privilégié d'une République méritocratique, elle apparaît comme un lieu éminemment démocratique. L'école semble détenir à elle seule la clé de l'avenir. Lorsque, deux ans après la Libération, le plan Langevin-Wallon voit le jour, cette utopie éducative, vivifiée par les idées de la Résistance ${ }^{3}$, porte à sa perfection l'idéal de l'égalité des chances dont les instituteurs sont les garants incorruptibles.

Qui dit mérite dit effort. L'école primaire des années 1930 et 1950 est essentiellement un lieu de travail dont l'austérité n'est pas absente. Elle requiert chez l'enfant de la bonne volonté et de la ténacité, et aussi, parfois, une pointe d'héroïsme. À cet égard le chemin de l'école constitue pour les écoliers une véritable mise à l'épreuve. Livrés à eux-mêmes, les petits ruraux doivent braver le froid, la neige et le vent et ce trajet, à la limite, prend des allures de chemin de croix :

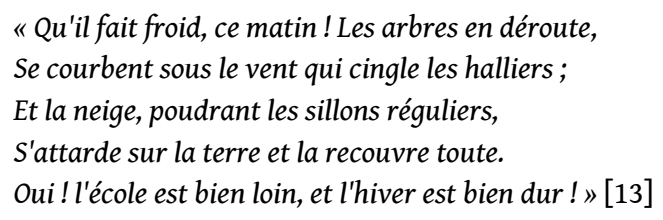

Par contraste avec la rudesse du milieu ambiant, l'école apparaît confortable et fonctionnelle. La vaste maison grise aux larges fenêtres est bien chauffée. Elle est aussi parfaitement hygiénique. Tout reluit, tout brille. De beaux livres, des cahiers neufs 
reposent sur les pupitres, pour servir le désir d'apprendre des enfants parmi lesquels règne une convivialité de bon aloi.

\subsection{Une instance de socialisation} idéalement organisée. Elle est le lieu où se révèle enfin la notion de patrie, dont tous les écoliers de France sont les enfants. Cette patrie - qui se confond souvent avec la République - ils doivent la "préférer à eux-mêmes, à leurs intérêts, à leurs propres familles" pour les bienfaits qu'elle leur octroie généreusement. Les enfants ont une dette envers elle, dont ils devront s'acquitter ultérieurement, par leur comportement de bons citoyens ${ }^{5}$ et, le cas échéant, de soldats valeureux. L'écolier est d'ailleurs souvent comparé à un conscrit prêt à accomplir son service militaire et fier de se battre pour son pays, et l'école assimilée à la mère patrie : «Comme il est beau cet héroïsme qui s'ignore et qui sait oublier le danger quand il faut songer qu'au devoir " ${ }^{1}$.Ce sont ces mâles sentiments qui nourrissent précocement une confiance forte en l'avenir :

\footnotetext{
«Marchez, pourtant, marchez d'un pas vaillant et sûr, Enfants, vers le devoir, le travail, l'espérance... Chacun, pour le pays, doit peiner à son tour... Marchez vers le savoir, car vous serez un jour, Humbles petits cerveaux, le cerveau de la France! " [19]
}

\subsection{L'école autarcique}

Toutes ces remarques s'appliquent - à quelques détails près - aux représentations scolaires qui émaillent les manuels des années 1930 et 1950.

Cependant, dans les années 1980, cet univers change de nature. Dans les ouvrages les plus récents, l'école primaire dépasse le niveau de l'utile pour atteindre l'agréable. Mieux, elle semble désormais conçue pour le service des enfants et non pour le service de la République. 
31 Cela se sent à des détails matériels. Le chauffage électrique remplace désormais l'antique poêle à charbon autour duquel les petits élèves en tablier noir se penchaient sur leurs pupitres pour recopier à la plume leurs exercices écrits. La salle de classe se fait plus amène, plus colorée, moins géométrique, et l'on dirait que les enfants passent plus de temps à jouer dans la cour de récréation qu'assis à leurs pupitres.

Certes, on trouve ici et là quelques survivances du passé. Ainsi un instituteur vérifie parfois que les écoliers conservent leurs mains et leurs ongles propres. Plus généralement, les enfants doivent toujours faire preuve d'une certaine discipline, être en mesure d'écouter et ils sont tenus de répondre correctement aux maîtres qui les interrogent. Mais, si le calcul semble toujours au programme des études, les apprentissages fondamentaux de la lecture et de l'écriture n'apparaissent pas dans les manuels récents que nous avons consultés, comme si ces savoirs allaient de soi, comme si l'école n'était plus aussi indispensable qu'autrefois.

Parallèlement l'école cesse d'être le lieu où les enfants acquièrent la compréhension du sens de la vie. D'ailleurs, elle n'est plus présentée comme une seconde famille et, de ce fait, semble avoir perdu de son aura ancienne. Les parents n'apparaissent que très rarement, juste dans une relation de suivi scolaire avec le directeur de l'école.

\section{Les personnages}

\subsection{Maîtres et maîtresses}

Au total, le maître est assez peu représenté dans notre corpus. Ses caractères sont d'ailleurs assez flous: on note simplement qu'il est rarement vêtu de la blouse grise traditionnelle - vraisemblablement parce que des habits bourgeois confèrent à sa fonction un surcroît de dignité - et qu'il paraît âgé, avec ses cheveux blancs, sans doute parce que l'âge est le plus sûr symptôme du savoir et de la sagesse. Pour un peu, on pourrait le soupçonner de manquer de personnalité, ce qui peut être interprété positivement comme un excès de conscience professionnelle : dans la salle de classe, l'homme s'efface devant sa mission ce qui lui donne parfois un abord un peu bourru :

"Le maître, dis maman, il ne me connaît pas?

Mais si, répond la mère avec un fin sourire;

C'est un autre papa qui va t'apprendre à lire.

L'école est sa famille: ainsi chaque écolier

Est un enfant qu'il aime avec son cour entier. » [1]

Les leçons de l'instituteur sont magistrales et irremplaçables. Malheur à l'enfant qui ne profite pas de sa parole infaillible, car «l'ignorance est une honte et un danger » [1]. Les livres du Cours Élémentaire regorgent d'anecdotes édifiantes illustrant les bienfaits de l'instruction, comme l'histoire du petit Thomas, qui, ne sachant pas lire, empoisonne son père malade en se trompant de fioles : l'une contenait du sirop et l'autre une sorte d'huile noirâtre avec laquelle le père devait se frotter le corps ${ }^{6}$.

Si la salle de classe est l'horizon du maître, la cour de récréation, si l'on en croit les manuels, est le royaume de la maitresse. Elle est globalement plus jeune que son collègue masculin, plus souriante aussi. Avec ses habits de couleurs, elle évince inexorablement le vieux maître d'école sévèrement vêtu. Sa pédagogie est d'ailleurs différente: moins raisonneuse, plus dynamique et affective, elle découle en quelque sorte de ces qualités et de ces apparences. Si l'on trouve exceptionnellement dans un des ouvrages consultés, un 
portrait de maîtresse grognon, à l'ancienne, on voit que l'institutrice moderne a le don d'entraîner littéralement les enfants à sa suite au point que, subjugués, certains la comparent parfois à une fée.

\subsection{Ecoliers et écolières}

Dans les années 1930 et 1950, le contrat pédagogique est clairement énoncé : les enfants s'engagent auprès de leurs maîtres à bien travailler. Ce pacte est basé sur un rapport d'autorité et d'obéissance dont la baguette et la règle sont les gages, et sur une relation de confiance et de reconnaissance. L'éducation républicaine repose en effet sur une étroite solidarité : les élèves, quels qu'ils soient, s'interpellent, se disent bonjour et rivalisent d'affection et de prévenance envers leurs maîtres et leurs maîtresses qui représentent à leurs yeux un modèle respecté. Ce consensus est tel que ces écoliers ne se distinguent guère que par leur prénom.

Leur typologie est d'ailleurs des plus rudimentaires: les filles douces, timides, parfois timorées s'opposent aux garçons plus courageux et plus turbulents. Et - comme jadis à l'école d'Aix-la-Chapelle visitée par Charlemagne - les bons élèves s'opposent aux mauvais écoliers.

Cette simplicité antithétique est une commodité pédagogique : en effet, à ces époques, la transmission des valeurs scolaires et sociales passe plus par l'exemple vivant - à suivre ou à rejeter - que par un discours magistral théorique.

Cependant, dans les années 1980, ce socle moral s'effrite. Avec l'effacement progressif du maître et des parents, les enfants se retrouvent de plus en plus souvent seuls, livrés à euxmêmes, sans modèles imposés, avec pour guide leur imagination qui inspire leurs jeux débridés. C'est à eux, à partir de leurs expériences propres, qu'il appartient de construire le savoir...

A cette date, il y bien longtemps que la journée de l'écolier français ne commence plus par une leçon de morale...

Figure 4

La figure charismatique de l'instituteur.

image

LELU A., KUBLER L. \& VELTZEL L. (inspecteurs de l'enseignement primaire) : Vocabulaire Grammaire Orthographe et Composition Française, cours élémentaire, classes de 10e et 9e. Paris, Hatier, 1960, 16e édition. 
En rentrant de l'école, Nicole et Victor se consacrent à leurs devoirs. Figure 5a: Le personnage de Nicole.

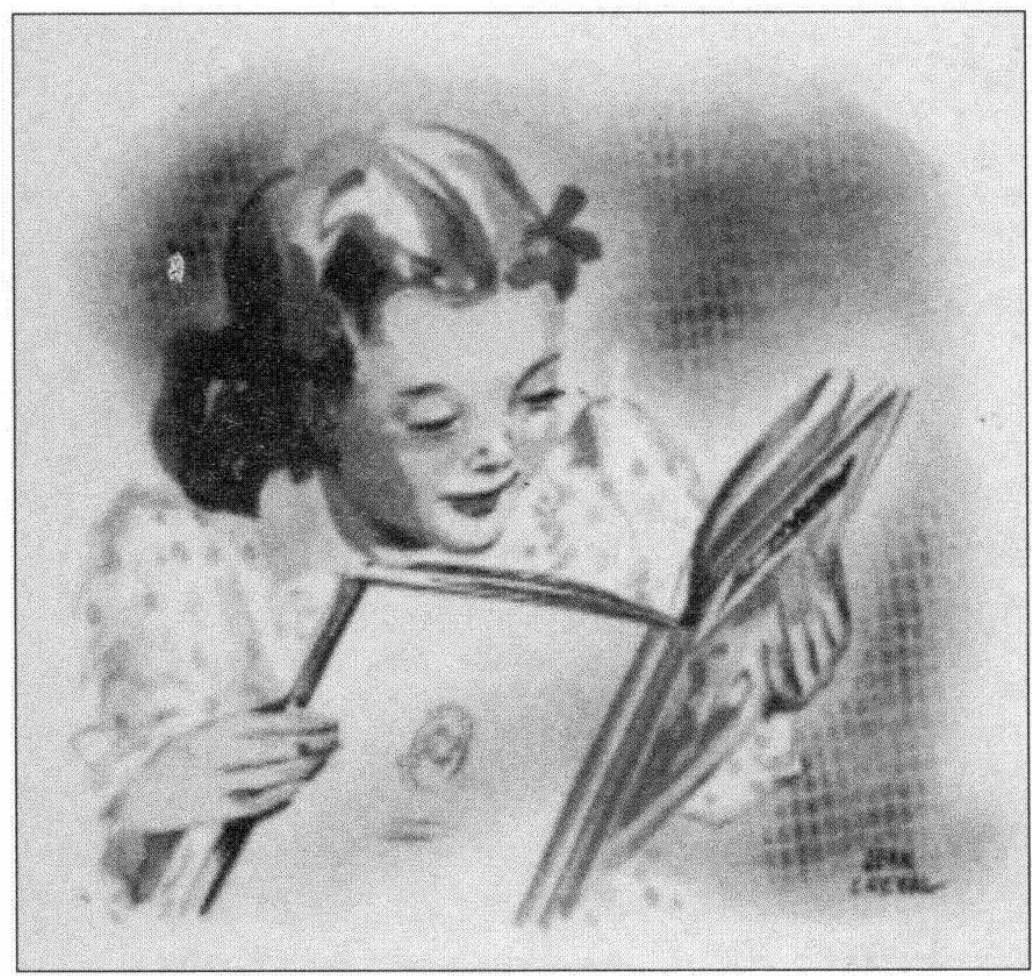

Figure $5 b$ : Le personnage de Victor.

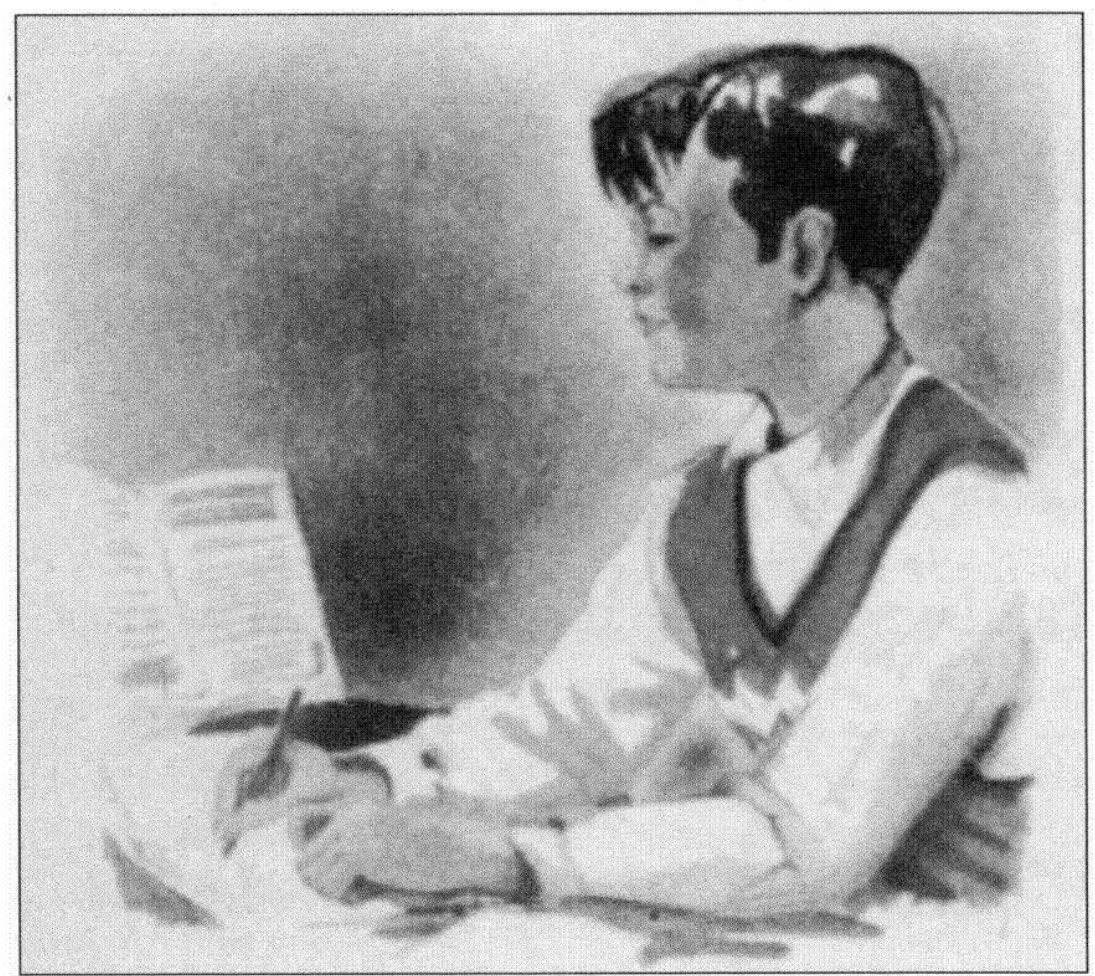

(Figures 5a et 5b) MILLET A. : Contes et Jeux de Nicole et Victor-Premier livre de lecture courante, Cours préparatoire, Cours élémentaire $7^{\text {re }}$ année. Paris, Larousse, 1948, 119 p., pp 76 et 82. 


\section{BIBLIOGRAPHIE}

\section{Corpus bibliographique}

\section{Années 1930}

[1] MIRONNEAU A. : Choix de lectures. Paris, A. Colin, 1930, 232 p.

[2] POMOT H., BESSEIGE H. et FOUROT A. : Pour bien lire. Paris, P.U.F, 1930, 288 p.

[3] AB DeR HALDEN Ch. et LAVAUT M. : La chaumine. Paris, Bourrelier, 1932, 191 p.

[4]POMOT H. et BESSEIGE H. : Pour bien lire - Ri, Guite et compagnie. Paris, P.U.F, 1932, 163 p.

[5] SEGUIN K. : En route pour l'école. Paris, Hachette, 1933, 183 p.

[6] LIQUIER P. : Heureuse enfance. Paris, Librairie L. Martinet, 1934, 227 p.

[7] JAUFFRÉ E. : Au pays bleu, roman d'une vie d'enfant. Paris, E. Belin, 1936, 255 p.

[8] BAUDRILLARD J. et KUHN M. : Lisons. Paris, F. Nathan, 1937, 223 p.

[8] AURIAC O., HAVARD H. et JUGHON B. : Nouveaux textes de lecture. Paris, A. Colin, 1939, 220 p.

\section{Années 1950}

[10] MAUGUIN E. et LEVANO H. : Histoire de Bouzou, le petit enfant d'ours. Paris, Hachette, 1950, $113 \mathrm{p}$.

[11]VIÉ A. : Quatre pas dans les champs. Paris, Hatier, 1951, 159 p.

[12]DURU G. et DURU M. : Lectures actives. Paris, Hachette, 1952, 191 p.

[13] NOUUÉ J. et ABRAHAM E. : Les textes littéraires. Paris, Limoges, Nancy, Charles-Lavauzelle et $\mathrm{C}^{\mathrm{ie}}, 1952,242 \mathrm{p}$.

[14] BRÉANT L.-M. et THIERRY B. : Nous les enfants. Paris, A. Colin, 1955, 159 p.

[15] DARDOISE M. : Le petit monde. Paris, F. Nathan, 1955, 159 p.

[16] BRANGIER L. et BALLEREAU E. : Les textes vivants. Paris, S.U.D.E.L., 1955, 224 p.

[17] POURON M., PICARD M ${ }^{\text {me }}$ et LEROY E. : Choix de lectures. Paris, Ch. Delagrave, 1956, 192 p.

[18] ROUSSEL M. : Quinze histoires de Gros-Pia. Paris, E. Belin, 1957, 189 p.

[19] NOUGUÉ J. et ABRAHAM E. : Les textes littéraires - Lecture et récitation. Paris, Limoges, Nancy, Charles-Lavauzelle, 1958, 130 p.

[20] ADENIS A. et COUSTEIX P. : Clairs sentiers. Paris, Strasbourg, Istra, 1959, 236 p.

[21] ROUSSEL M. : Fredi, Suzette et le canard boiteux. Paris, E. Belin, 1959, 127 p. 


\section{Années 1980}

[22] MAREUIL A. et HISBERGUE A. : Le livre des bêtes. Paris, Istra, 1980, 111 p.

[23] BEAUTÉ J. et al : Lalulapalu. Paris, Caref, Magnard, 1981, 100 p.

[24] GIRAUDIN G. et VIGO J. : L'oiseau-lyre. Paris, Hachette, 1981, 110 p.

[25] COTET G. : Vivre la vie rue des chats gris. Paris, Hachette, 1982, 64 p.

[26] LASCAR S. et LECLERC A. : Mon copain Bruno. Paris, Magnard, 1982, 59 p.

[27] MONTMAYEUR H., JOURDAIN D., FRANCKE L. et al : Le bateau livre. Paris, F. Nathan, 1983, 143 p.

[28] KERLOC'H J.-P. : La planète des livres. Paris, Bourrelier, A. Colin, 1984, 127 p.

[29] LELOUCH B. et THIRIOY J. : Horizon lecture. Paris, Ch. Delagrave, 1987, 127 p.

[30] CALLE V. et FERRAND L. : Je lis seul, tu lis seule. Paris, Nathan, 1989, Fiches.

[31] THOMAS J.-L., MASSANE J., LEMOINE J. et al : La ruche aux livres. Paris, Hachette, 1989, 157 p.

\section{NOTES}

1. Cette étude dérive d'un mémoire de DEA conduit sous la direction de Pierre Guibbert et soutenu en 1998 à l'université Paul Valéry de Montpellier.

2. Les chiffres entre crochets renvoient au corpus produit en fin d'article.

3. Sur ce sujet, $C f$. : Sous la direction de BOUTAN P. et SOREL E. : Le plan Langevin-Wallon, une utopie vivante. Paris, Presses Universitaires de France, Collection Éducation et Formation, 1998, $181 \mathrm{p}$.

4. STEEG J. : Le livre de morale du petit citoyen. Paris, F. Nathan, $1882,179 \mathrm{p}$.

5. Sur les manuels d'apprentissage de la morale à l'école primaire, $C f$.: GUIBBERT P. : «A l'école de la République ", in Les Mauvaises consciences françaises. Paris, Panoramiques, 1998, 208 p., pp. 110-120.

6. Cette historiette a été rapportée par : GUIBBERT P.: in «Entreprise moralisatrice et ruralité dans les manuels de la III ${ }^{\mathrm{e}}$ République ", in Tréma, No 12-13. Montpellier, I.U.F.M. de l'Académie de Montpellier et Université Paul Valéry, 1997, 192 p., pp. 125-141.

\section{RÉSUMÉS}

Non disponible

Not available 
INDEX

Mots-clés : écoliers, enseignement primaire, France, lecture, manuel scolaire, moralisation Keywords : primary school education, reading, school textbook, secondary school teaching

\section{AUTEUR}

NICOLE BOURDON

Université Paul Valéry Montpellier 